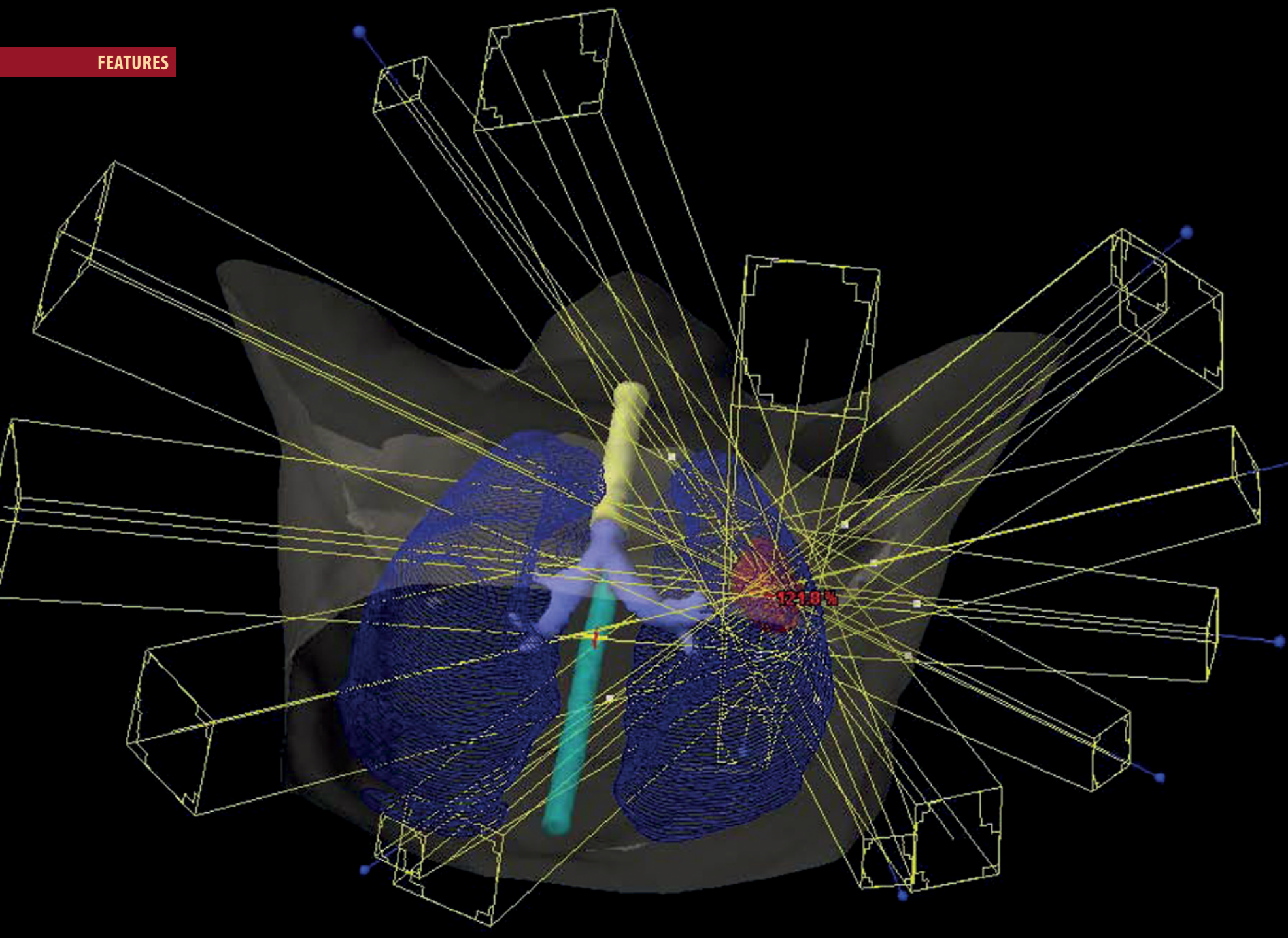

\title{
Advances in radiotherapy with external beam X-rays: from 3D to 4D
}

- Montserrat Ribas Morales - Hospital de la Santa Creu i Sant Pau, Barcelona, Spain - DOI: 10.1051/epn/2012605

Radiotherapy (RT) is the medical use of ionizing radiation, generally as part of cancer treatment to control or kill malignant cells. About 50 to $60 \%$ of all cancer patients in Europe receive RT either as curative therapy alone, or as an adjunct to surgery and/or chemotherapy, or as palliative therapy.

A Illustration of radiotherapy treatment in which the dose is concentrated on a small piece of lung tissue (so-called

stereotactic body radiation therapy) (Image from HSCSP) he goal of radiotherapy is to deliver a high dose of radiation to the tumour volume while keeping the dose to healthy tissue as low as possible. If the dose is too high the complication rate increases, but if it is too low the probability of tumour control decreases. This paper explains the process of RT, focusing on the current standard technique, which is called 3 -dimensional conformal radiation therapy (3D-CRT) using external-beam X-ray RT, and its technological improvements.

\section{External-beam X-ray RT}

In most centres today, external-beam radiation treatment is delivered to the patient by means of an electron linear accelerator, known as "linac". Linacs deliver radiation beams from different angles around the patient. These 
beams pass through the patient delivering the dose along their path. The combination of various shaped beams of radiation concentrates the dose in the tumor while surrounding healthy tissues receive the lowest possible dose. Treatment in RT involves multidisciplinary professionals such as radiation oncologists, medical physicists and technicians, and requires their close collaboration to be safe and effective. The process for treatment generally covers the following steps (see figure 1):

1. Dose prescription. The radiation oncologist prescribes the total dose of treatment to the tumour and the dose constraints to adjacent healthy organs, called organs at risk. The total treatment dose depends on the type of tumour. It usually varies from 40 to $80 \mathrm{~Gy}$ and is administered in several fractions, usually from 1.8 to $2 \mathrm{~Gy}$ per session. The number of sessions ranges from 20 to 40 , depending on the planned total dose. Overall treatment time is approximately 4 to 8 weeks.

2. Treatment planning. Treatment planning is currently based on 3D images taken using X-ray computed tomography $(\mathrm{CT})$. To obtain these images, patients are placed in the same position in which they will be treated daily in the linac, and the same immobilization devices are used. CT images have a high contrast resolution to discriminate between values of tissue densities that differ up to $0.1 \%$ and a high spatial resolution (on the order of 1-2 mm). They make possible to determine ('delineate') the volume to be irradiated and the organs at risk. When supplementary information is needed in regions of the body such as lung, head and neck, images are taken by magnetic resonance (MR) or positron emission tomography (PET) and combined with these $\mathrm{CT}$ images. PET has a high contrast resolution, but its anatomic resolution is limited. Using images of both PET and MR we can distinguish the most resistant areas in a tumour and treat these with higher doses. In general, PET and CT are used for planning in lung and head and neck cancers, while MR and CT are used in tumours such as those in the brain or prostate. By better defining the target volume with data acquired from PET or MR we can deliver a larger dose to the patient and reduce damage to surrounding healthy tissue.

\section{D CT imaging}

Respiratory motion is one of the major challenges in RT in thoracic and abdominal cancers. Movement of the tumor can lead to uncertainty in delineating the volume to be irradiated and therefore affect the treatment dose delivery. Conventional RT does not take this respiratory motion into account when planning the target volume. To compensate for this, a uniform margin is added to the tumour volume. Tumour motion is anisotropic and it varies from patient to patient. The magnitude of the movement depends not only on each patient's respiratory cycle but also on the size and location of the tumour. Displacement of tumour motion may vary significantly depending on the direction of the patient (superior-inferior, lateral and anterior-posterior) [1].

To analyse respiratory motion, $4 \mathrm{D} \mathrm{CT}$ imaging is used. 4D CT images can only be obtained with new-generation CT scanners. These machines have faster data acquisition and yield dynamic CT images of the thoracic region. $4 \mathrm{D}$ CT imaging collects time-varying scans of the lungs. The first step is to record the patient's respiratory cycle.4D CT imaging can only be used when patients have a regular and reproducible respiratory cycle. The breathing cycle is divided into distinct phases, and data sets of CT images are acquired for each phase and in every position of interest. After the scan is taken, images are sorted and correlated based on the corresponding breathing phase signals. Thus, many 3D CT sets are obtained, each corresponding to a specific breathing phase. Together, they constitute a 4D CT series of images that covers the whole breathing cycle. The radiation oncologist may choose to work on the images of a single phase or the images of the whole cycle. This $4 \mathrm{D}$ CT process allows the radiation oncologist to better delineate the target volume and reduce the volume of normal tissue to be irradiated.

Because 4D CT provides detailed visualization of the lung movements it implies considerable progress in treating tumors of the chest and upper abdomen.

3. Dose calculation. The dose contribution from each beam is calculated by a treatment-planning system using CT images of the patient and a mathematical model of how the linac will deliver doses in different tissues. The shape of each beam is controlled by collimators or multileaf collimators (MLC). Multi-leaf collimators consist of two sets of individual "leaves" (from 20 to 160 leaves per set) that can move independently in and out of the path of a beam to block it. One or several beams can be used, depending on the disease and the patient's needs.

V FIG. 1: External 3D-Conformal Radiation Therapy process

\section{External 3D-CRT process}

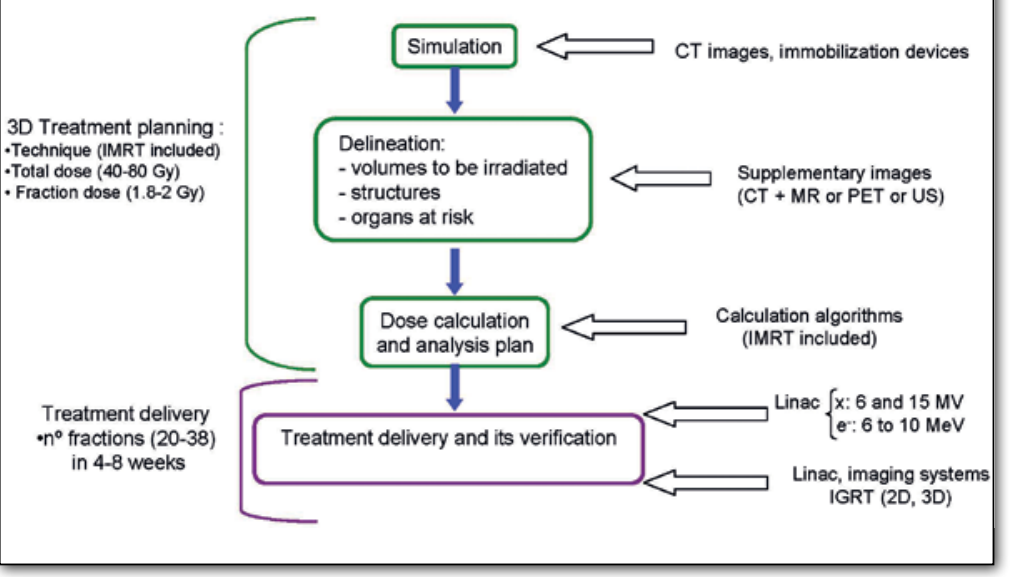


v FIG. 2: A snapshot of the multi-leaf collimators (MLC) during a head and neck treatment using dynamic intensity-modulated radiotherapy. The main collimator is shown as a yellow rectangle. The yellow lines show the start and final positions of the MLC. The blue lines show an instantaneous position of the MLC. (Image from HSCSP).
Some treatments need radiation fields that have non-uniform spatial intensity distributions. To obtain the desired dose distribution in the tumour target volume we need to combine several beams with a non-uniform dose so as to spare nearby critical structures. This procedure is called intensity-modulated radiotherapy (IMRT). IMRT is an advanced step of 3D CRT. IMRT uses multi-leaf collimators that can move dynamically during the treatment session when the beam is on, or between exposures. The process of dose calculation is the responsibility of the medical physicist. The radiation oncologists analyse the treatment plan and if they agree with this, it is approved and forwarded to the treatment unit for delivery (see figure 2).

4. Treatment delivery. The dose is delivered in a linac, as mentioned above. Linacs can be designed to produce monoenergetic photons with fixed energy (usually $6 \mathrm{MV}$ ), or multi-energetic photons ( 6 to $15 \mathrm{MV}$ ) and electrons (6 to $20 \mathrm{MeV}$ ). It is important that the radiation is delivered to the correct anatomic site. To verify the patient setup in the treatment position, traditional films have now been replaced by electronic portal imaging devices attached to the gantry of the linac and aligned with the beam.

3D CRT, intensity-modulated radiotherapy (IMRT) and other recently developed techniques have reduced the alignment error tolerance for targets that move and deform during treatment. However, these techniques are complex. To deal with these challenging treatment scenarios, linac manufacturers have introduced image-guided radiotherapy $3 \mathrm{D}$ and developed and integrated X-ray imaging systems (see figure 3) to improve and facilitate $3 \mathrm{D}$ visualization of the patient's internal anatomy. This enables efficient positioning of these anatomical structures in relation to the linac. Most of these systems use CT-based technology.

With these technological improvements, a new RT procedure has emerged, called stereotactic body radiation therapy. It is highly effective for small lesions $(<6 \mathrm{~cm})$ in the abdominopelvic and thoracic region. The main feature separating stereotactic body radiation therapy from standard 3D RT or IMRT treatment is that large doses $(6-30 \mathrm{~Gy})$ are delivered in a few fractions $(1-5)$ over a relatively short time ( 2 weeks), resulting in a high biological effective dose. To minimize normal tissue toxicity, it is essential to confine these high doses to the target and achieve rapid fall-off of the doses away from the target. So-called 'Stereotactic body radiation therapy' integrates simulation, treatment planning and dose administration and requires a high level of confidence in the accuracy [2] Due to the rapid adoption of stereotactic body radiation therapy and other modalities of IMRT, specific linacs have been developed, with single photon energy of $6 \mathrm{MV}$. These new linacs incorporate different systems of image-guided 3D depending on the manufacturer. They may be based on $\mathrm{CT}$, dual radiographic $\mathrm{x}$-ray imaging, and/or fluoroscopy.

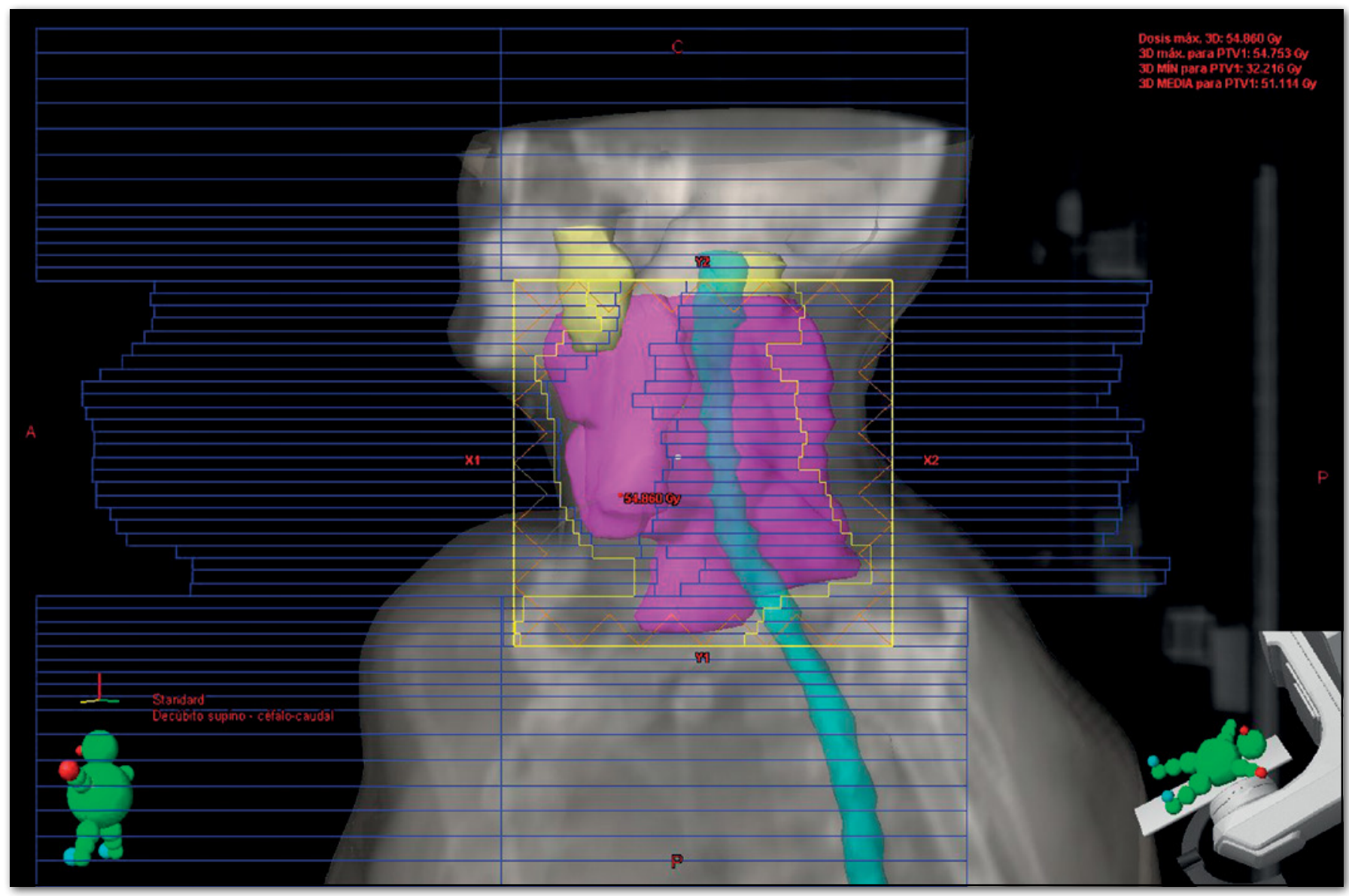




\section{D Treatment delivery}

$4 \mathrm{D}$ treatment delivery is analogous to $4 \mathrm{D} \mathrm{CT}$ imaging discussed earlier. It means that respiratory motion is taken into account during RT treatment. The recommendations to apply $4 \mathrm{D}$ in the treatment of patients with thoracic, abdominal, and pelvic tumours affected by respiratory motion can be summarized if either of the following conditions are met [3]: when a range of motion greater than $5 \mathrm{~mm}$ is observed in any direction; or when significant normal tissue sparing can be gained through the use of a respiration management technique.

There are several methods to reduce the impact of respiratory motion in RT, but one of the most widely accepted modes by the radiation oncologists is real-time tumour tracking. Under ideal conditions, tracking can eliminate the need for a tumour-motion margin in the dose distribution, while maintaining a $100 \%$ duty cycle for dose delivery. For this tracking to be successful, four criteria must be met:

1. the tumour position must be identified in real time;

2. tumour motion must be anticipated to allow for time delays in the response of the beam-positioning system;

3. the beam must be repositioned;

4. dosimetry must be adapted to allow changes in lung volume and the location of critical organs at risk during the breathing cycle.

Only a few brands of linacs currently offer tracking. Research is ongoing in the field to improve or incorporate "tracking" in the linacs, adjusting the dynamic movements of collimators or multi-leaf collimators.

As management of respiratory motion requires the use of highly specialized technology it is recommended that a qualified medical physicist be present throughout the entire process for each patient, from the treatment-planning imaging sessions to treatment delivery.

\section{Adaptive RT}

Adaptive RT is the optimization of treatment planning based on information acquired during the course of fractionated treatment. RT is a dynamic process. Patient outcome depends on four variables: the location of the dose in each session; the variation of dose administration over time; the anatomical geometry of the tumour; and, the biological response of the tumour and normal tissue. As advances in RT allow a higher dose of RT to the tumour and lower dose to organs at risk, correct patient alignment and knowledge of changing internal anatomy is critical.

Until recently, all treatment was planned before the first fraction was delivered to the patient, and no further changes were made throughout the course of therapy. However, a new concept is now being introduced as a result of advances in current technology. Adaptive radiotherapy (ART), also called adaptive radiotherapy $4 \mathrm{D}$, is

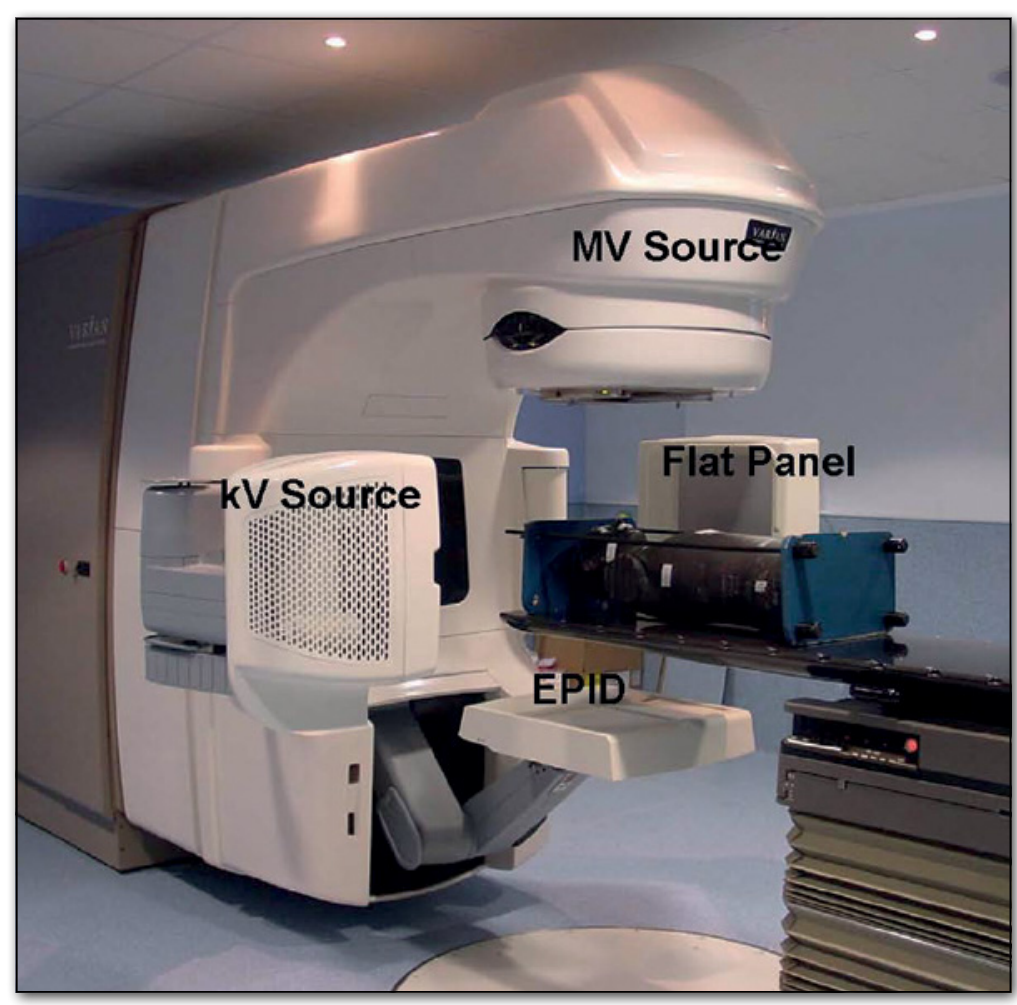

the explicit inclusion of the temporal changes in anatomy during the imaging, planning, and delivery of radiotherapy [4]. Adaptive radiotherapy $4 \mathrm{D}$ allows the radiation oncologist to modify treatment in cases of weight loss, tumour shrinkage or growth, or volumetric or dosimetric changes in the tumour. The tools and methods to perform such modifications are under continuous development and present results strongly suggest that ART will replace 3D CRT in the near future.

\section{About the Author}

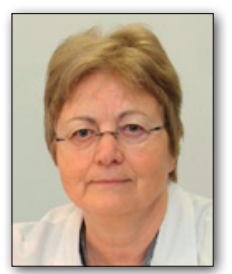

Montserrat Ribas Morales studied Physics at the University of Barcelona (Spain). PhD in Physics. Specialist in Medical Physics (Radiofísica Hospitalaria in Spain). Since 1972 works at Hospital Santa Creu i Sant Pau (Barcelona, Spain). Currently she is the Head of Medical Physics Department (Servei de Radiofísica i Radioproteccio) in this hospital. Since 2005 chair of National Comission of "Radiofisica Hospitalaria", consulting body of Ministry of Health.

\section{References}

[1] H.Helen Liu and colls, Int. J. Radiation Oncology Biol. Phys. 68-2, 531 (2007).

[2] AAPM. Stereotactic body radiation therapy: The report of AAPM Task Group 111. Med.Phys. 37-8, (2010).

[3] AAPM Report n. 91. The management of respiratory motion in radiation oncology, AAPM TG 76, (2006).

[4] http://medicalphysicsweb.org $\triangle$ FIG. 3: Linac with integrated X-ray imaging systems. (Image from HSCSP). 